\title{
DEPRESSION AND ANXIETY IN HASHIMOTO THYROIDITIS AFTER LONG-TERM LEVOTHYROXINE REPLACEMENT
}

\author{
Djurović M. ${ }^{1}$, Pereira AM², Jemuović Z.1, Vasović 0. ${ }^{3}$, Pavlovic D.4, Petakov M.1, Milfic D. ${ }^{1}$, Damjanovic S. \\ ${ }^{1}$ Clinic for Endocrinology, Diabetes and Diseases of Metabolism, Clinical Centre of Serbia, University of Belgrade, Belgrade, Serbia. \\ ${ }^{2}$ Department of Medicine, Division of Endocrinology, Leiden University Medical Center, Leiden, The Netherlands. \\ 3 Institute for gerontology and palliative care, Belgrade, Serbia \\ ${ }^{4}$ Faculty of philosophy, University of Belgrade, Belgrade, Serbia.
}

Introduction: Thyroid dysfunction may cause various psychiatric symptoms and disturbances. In addition, many factors affect the variable responses to hormone replacement. Many patients treated for Hashimoto thyroiditis (HT) report persistent impairments in general well-being despite long-term L-thyroxine (LT4) replacement and restoration of biochemical euthyroidism.

Aim: to investigate the prevalence of depression and anxiety in patients with HT on long-term LT4 replacement and potential associations with free T4 (FT4), TSH, and antithyroid peroxidase antibodies (anti-TPO) concentrations.
Subjects and Methods: cross-sectional, case-control study with 120 patients with HT on long-term LT4 replacement and 60 euthyroid control subjects, matched for age, gender, and educational level. Measurement of TSH, FT4 and anti-TPO, and evaluation of depression and anxiety using the Hospital Anxiety and Depression Scale (HADS). Patients and controls were further subdivided according to age: group $A$ (20-49 yrs) and $B$ (50-75 yrs). Fisher's ANOVA analysis was used to compare means between (sub)groups, Spearman's correlation coefficient(r) to assess the correlations between hormone levels, anti-TPO, and HADS scores.

\section{Results:}

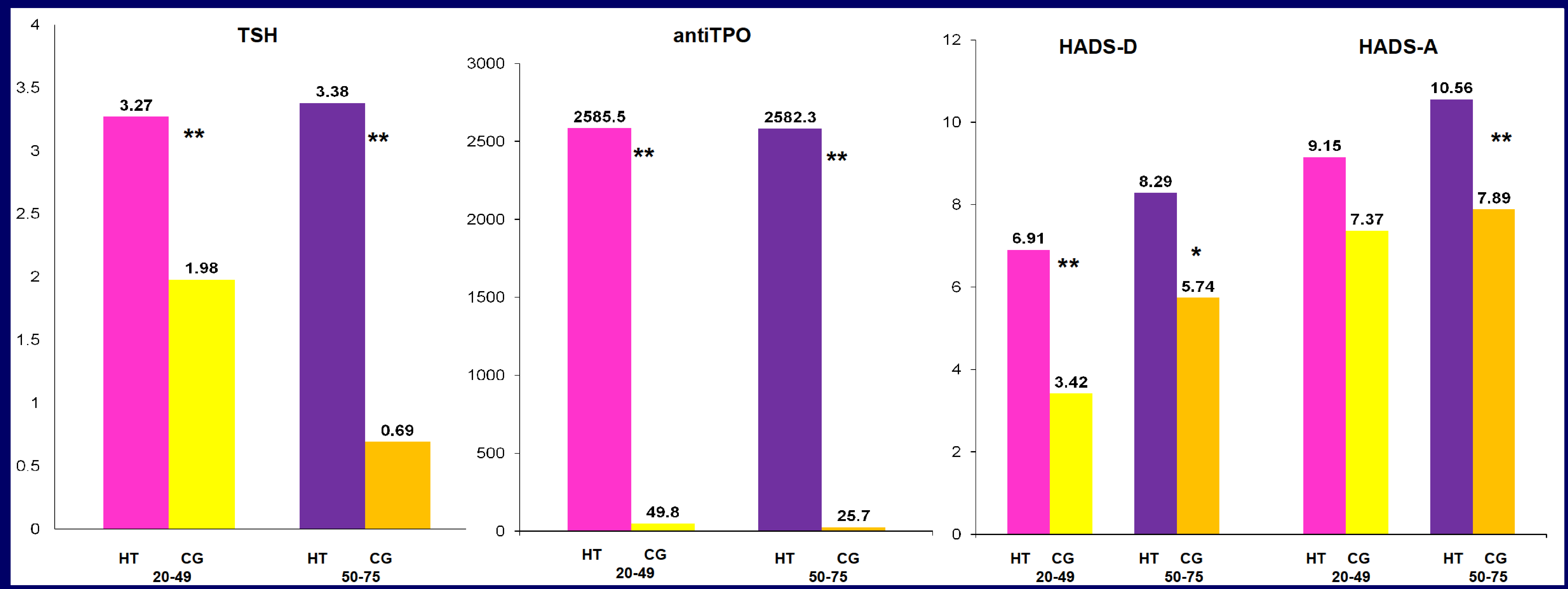

${ }^{* *} p<0.01$

${ }^{*} p<0.05$

\begin{tabular}{|l|l|l|}
\hline & HADS-D & HADS-A \\
\hline Disease duration & $r=0.418, \quad p<0.001^{* *}$ & $r=0.292, \quad p<0.001^{* *}$ \\
\hline duration of LT4 replacement & $r=0.221, \quad p=0.019^{*}$ & $r=0.053, \quad p=0.584$ \\
\hline TSH & $r=0.246, \quad p=0.001^{* *}$ & $r=0.202, \quad p=0.005^{* *}$ \\
\hline antiTPO & $r=0.170, \quad p=0.021^{*}$ & $r=0.169, \quad p=0.022^{*}$ \\
\hline FT4 & $r=-0.156, p=0.030^{*}$ & $r=-0.049, \quad p=0.494$ \\
\hline FT3 & $r=-0.166, p=0.035^{*}$ & $r=-0.077, \quad p=0.328$ \\
\hline
\end{tabular}

Conclusion: patients on long-term levothyroxine replacement for HT have more anxiety and depression. These symptoms associate both with the duration of treatment and with higher anti-TPO concentrations. 